\title{
Experimental investigation of transverse flow estimation using transverse oscillation
}

\author{
Udesen, Jesper; Jensen, Jørgen Arendt
}

Published in:

2003 IEEE International Ultrasonics Symposium Proceedings

Link to article, DOI:

10.1109/ULTSYM.2003.1293211

Publication date:

2003

Document Version

Publisher's PDF, also known as Version of record

Link back to DTU Orbit

Citation (APA):

Udesen, J., \& Jensen, J. A. (2003). Experimental investigation of transverse flow estimation using transverse oscillation. In 2003 IEEE International Ultrasonics Symposium Proceedings (pp. 1586-1589). IEEE. I E E E International Ultrasonics Symposium. Proceedings https://doi.org/10.1109/ULTSYM.2003.1293211

\section{General rights}

Copyright and moral rights for the publications made accessible in the public portal are retained by the authors and/or other copyright owners and it is a condition of accessing publications that users recognise and abide by the legal requirements associated with these rights.

- Users may download and print one copy of any publication from the public portal for the purpose of private study or research

- You may not further distribute the material or use it for any profit-making activity or commercial gain

- You may freely distribute the URL identifying the publication in the public portal 


\title{
Experimental Investigation of Transverse Flow Estimation using Transverse Oscillation
}

\author{
Jesper Udesen ${ }^{1,2}$ and Jørgen Arendt Jensen ${ }^{1}$ \\ 1) Center for Fast Ultrasound Imaging, Ørsted•DTU, \\ Bldg. 348, Technical University of Denmark, DK-2800 Lyngby, Denmark. \\ 2) B-K Medical A/S, Mileparken 34, DK-2730 Herlev, Denmark.
}

\begin{abstract}
Conventional ultrasound scanners can only display the blood velocity component parallel to the ultrasound beam. Introducing a laterally oscillating field gives signals from which the transverse velocity component can be estimated using 2:1 parallel receive beamformers. To yield the performance of the approach, this paper presents simulated and experimental results, obtained at a blood velocity angle transverse to the ultrasound beam.
\end{abstract}

The Field II program is used to simulate a setup with a 128 element linear array transducer. At a depth of $27 \mathrm{~mm}$ a virtual blood vessel of radius $2.4 \mathrm{~mm}$ is situated perpendicular to the ultrasound beam. The velocity profile of the blood is parabolic, and the speed of the blood in the center of the vessel is $1.1 \mathrm{~m} / \mathrm{s}$. An extended autocorrelation algorithm is used for velocity estimation for 310 trials, each containing 32 beamformed signals. The velocity can be estimated with a mean relative bias of $6.3 \%$ and a mean relative standard deviation of $5.4 \%$ over the entire vessel length.

With the experimental ultrasound scanner RASMUS the simulations are reproduced in a experimental flow phantom using a linear array transducer and vessel characteristics as in the simulations. The flow is generated with the Compuflow 1000 programmable flow pump giving a parabolic velocity profile of the blood mimicking fluid in the flow phantom. The profiles are estimated for 310 trials each containing of 32 data vectors. The relative mean bias over the entire blood vessel is found to be $10.0 \%$ and the relative mean standard deviation is found to be $9.8 \%$.

With the Compuflow1000 programmable flow pump a color flow mode image is produced of the experimental setup for a parabolic flow. Also the flow of the human femoralis is reproduced and it is found that the characteristics of the flow can be estimated.

\section{INTRODUCTION}

In conventional ultrasound scanners the point spread function (PSF) only has an axial oscillation, and hence blood scatterers travelling through the acoustic field only gives signals from which the axial velocity of the blood can be determined. This is a severe limitation, since most blood vessels are parallel to the skin surface, and it is therefore a problem to achieve a sufficiently small angle between the flow and the beam. One way to overcome this problem is the introduction of two PSF's each with an oscillation both in the axial and in the lateral direction and each PSF transverse phase shifted $90^{\circ}$ from each other (Fig. 2). This method has been suggested both by Jensen and Munk (Transverse Oscillation (TO)) [1, 2, 3] and by Anderson (Heterodyned Spatial Quadrature (HSQ)) [4, 5]. The major differences between the two methods is the way the two PSF's are constructed. Anderson is using two different apodizations in receive, where Jensen and Munk are using axicon focus in receive to create the $90^{\circ}$ phase modulation. Furthermore the applied velocity estimators are different.

This paper investigates the performance of the TO method. The performance is determined using the ultrasound simulation program Field II [6], and phantom experiments using an experimental scanner and a flow phantom attached to a flow pump.

\section{Simulated SETUP AND RESUlTS}

The Field II program is used to simulate a 128 element linear array transducer and a cylindrical blood vessel perpendicular to the beam direction. The acoustic field emitted from the transducer resembles that of a conventional Doppler system i.e hamming apodization and quadratic focusing is applied. The received signals are beamformed using axicon focusing and two sinc functions as apodization (see Fig. 1). This gives a PSF with an oscillation in both the axial and transverse direction. To have two PSF's, which are $90^{\circ}$ transverse phase shifted from each other, two beamformers are applied, and the $90^{\circ}$ phase modulation is created with two different axicon focuses. The exact setup parameters can be found in Table 1 and Table 2 and a detailed description of the theory for the transducer setup and the beamforming can be found in [2]. 
The corresponding PSF's can be seen in Fig. 2.

To make the simulation as simple as possible, and hence decrease computation time, no tissue was introduced around the vessel i.e. the only scatterers present in the simulation were the blood scatterers. They were moved transverse to the beam direction with a parabolic velocity profile $v$ given by $v=v_{0}\left(1-\left(\frac{r}{R}\right)^{2}\right)$, where $R$ is the radius of the vessel, $r$ is the distance from the axis of the vessel, and $v_{0}$ is the maximum velocity. Since the vessel had a finite length, scatterers passing the end of the vessel were introduced in the other end.
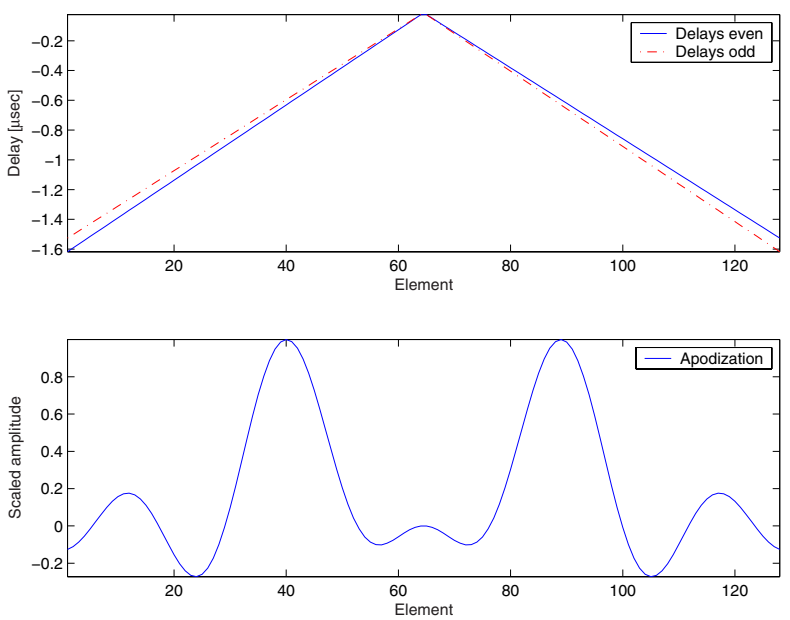

Figure 1: The delay times (top) and the apodization (bottom) used for the beamforming.
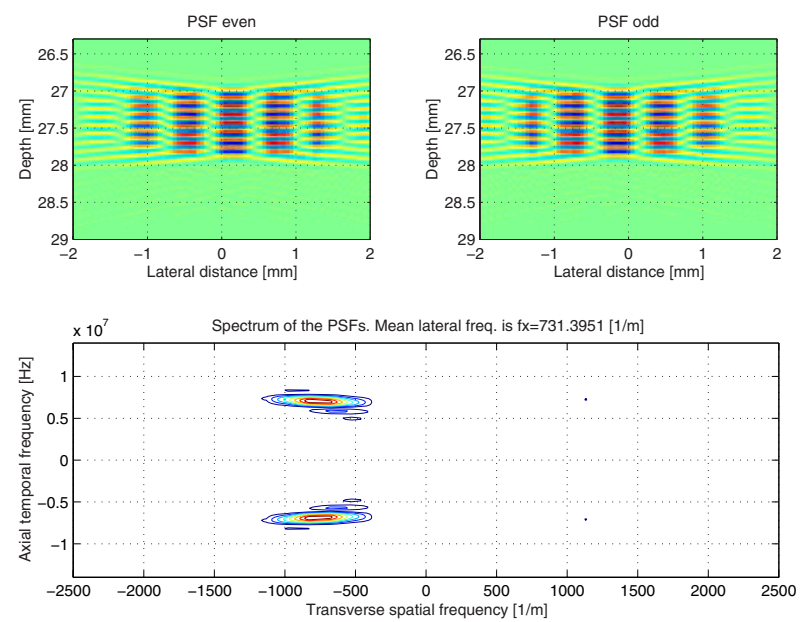

Figure 2: The two PSF's used for transverse velocity estimation and their corresponding spectra. The point scatterer is situated at a depth of $27 \mathrm{~mm}$.

The velocity estimation was performed using an extended

\begin{tabular}{|l|l|}
\hline Transducer & Linear array \\
Number of elements & 128 \\
Pitch & $0.208 \mathrm{~mm}$ \\
Kerf & $0.035 \mathrm{~mm}$ \\
Center frequency & $7 \mathrm{MHz}$ \\
Number of cycles pr. pulse & 8 \\
fprf & $8 \mathrm{kHz}$ \\
Sampling frequency & $100 \mathrm{MHz}$ \\
Transverse wave length & $1.2 \mathrm{~mm}$ \\
Apodization in transmit & Hamming \\
Apodization in receive & Two sinc's \\
Focus in transmit & $54 \mathrm{~mm}$ \\
Focus in receive & Axicon at $27 \mathrm{~mm}$ \\
\hline
\end{tabular}

Table 1: Transducer setup

\begin{tabular}{|l|l|}
\hline Depth to vessel & $27 \mathrm{~mm}$ \\
Radius of vessel & $2.4 \mathrm{~mm}$ \\
Length of vessel & $20 \mathrm{~mm}$ \\
Number of scatterers & 12,387 \\
Maximum blood velocity & $1.1 \mathrm{~m} / \mathrm{s}$ \\
Blood velocity & Parabolic \\
\hline
\end{tabular}

Table 2: Parameters for the simulated blood vessel.

autocorrelation algorithm for lag 1 described in [3]. For standard deviation estimation 310 data ensembles were used each containing 32 beamformed received signals. Echo canceling was applied using a highpass filter with a cut off frequency at $f=80 \mathrm{~Hz}$ and $\mathrm{RF}$ averaging over one pulse length was applied.

Since the velocity is scaled with the lateral oscillation length $\lambda_{x}$, a correct value for $\lambda_{x}$ is desirable. In theory $\lambda_{x}$ can be derived from the Fraunhofer approximation to be $\lambda_{x}=z /\left(\lambda_{z} \xi\right)$, where $\lambda_{z}$ is the axial wave length, $z$ is the depth and $\xi$ is the spatial distance between the peaks of the two sinc functions used for the receive apodization $[2,7]$. Since the Fraunhofer approximation is based on a number of assumptions, which are only approximately satisfied, a more correct estimate for $\lambda_{x}$ can be derived using the PSF's for the transducer setup. Taking a 2D FFT of the PSF's and thereafter finding the mean value of the transverse frequencies gives a more correct value for $\lambda_{x}$, which then can be used in the velocity estimator. Typical the deviation between the theoretical value for $\lambda_{x}$ and the one derived from the PSF's is around $10 \%$. The resulting mean transverse velocity profile with corresponding standard deviations can be seen in Fig. 3. The velocity deviates from the theoretical velocity profile with a mean relative bias of $6.3 \%$ and a relative mean standard deviation of $5.4 \%$, taken over the entire vessel profile relative to the maximum blood velocity. Near the vessel wall there is a significant deviation between the velocity estimate and the theoretical parabolic profile. This is because the PSF's 


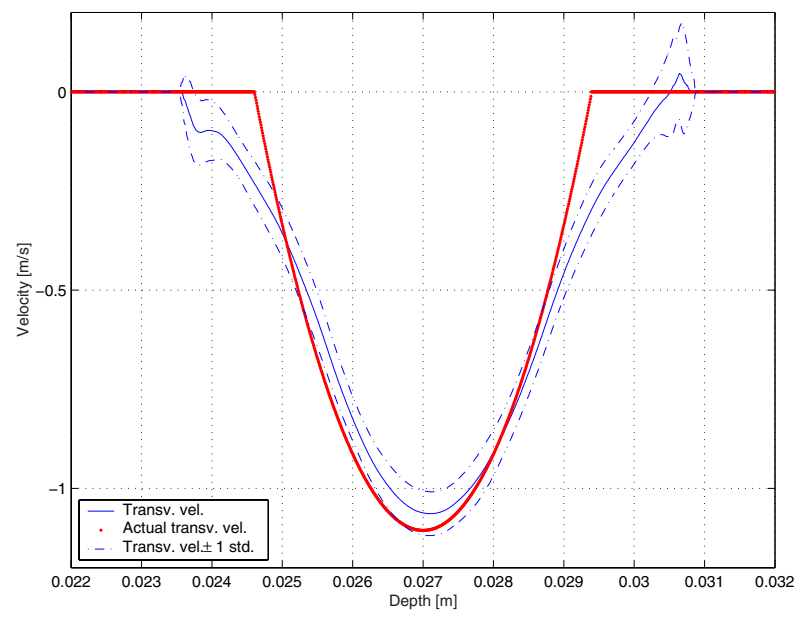

Figure 3: The mean velocity profile and corresponding standard deviation for the simulation using Field II.

have an axial extend of approximately $1 \mathrm{~mm}$, (see Fig. 2), and since every velocity estimate is an average over the velocities present inside the area of the PSF, the estimates will be "smooth" near the vessel walls.

\section{EXPERIMENTAL SETUP AND RESULTS}

A commercial linear array transducer with 128 elements was used to scan a flow phantom (SMI140 from Shelley Medical Imaging Technologies, London, Ontario, Canada) with blood mimicking fluid attached to a Shelley Compuflow1000 flow pump. The same setup parameters was used as in the simulation (see Table 1 and Table 2) except that matched filtering was performed on the acquired RF data to reduce noise. Furthermore the sampling frequency was decreased to $40 \mathrm{MHz}$, and tissue was present between the transducer and the vessel. The attenuation of the tissue was $0.7 \mathrm{~dB} / \mathrm{cm} / \mathrm{MHz}$ at 5 $\mathrm{MHz}$ and the blood mimicking fluid is described in [8]. The Reynolds number for the flow was approximately $10^{3}$, hence the flow can be regarded as laminar with a parabolic velocity profile similar to the one in the simulation.

The scanner used for data acquisition was the experimental scanner RASMUS [9], which has 128 channels in transmit and 64 channels in receive. To acquire 128 elements in receive, two consecutive received data sets was sampled, respectively on the left hand side and the right hand side of the transducer and thereafter superimposed to each other (see Fig. 5). Again 310 data ensembles were acquired each containing 32 beamformed signals. The same echo canceling filter and velocity estimator was used as in the simulation, and the resulting mean velocity profile can be seen in Fig. 4. The velocity deviates from the theoretical velocity profile with a relative mean bias of $10.0 \%$ and a relative mean standard de-

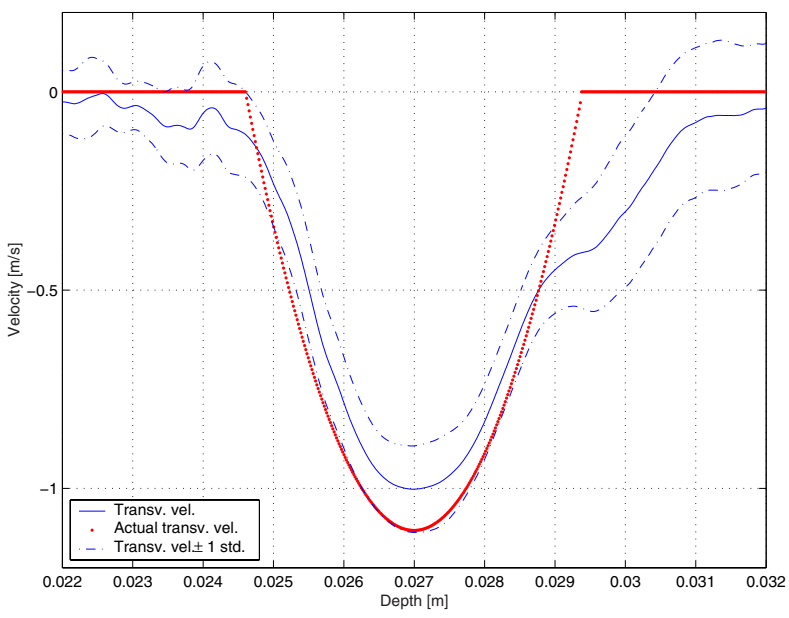

Figure 4: The mean velocity profile and corresponding standard deviation for the flow phantom experiment.

viation of $9.8 \%$ taken over the entire vessel profile, where the values are relative to the maximum velocity. As mentioned in the previous section the velocity is scaled with $\lambda_{x}$, and hence a wrong choice of $\lambda_{x}$ will result in a biased estimate. Therefore we believe, that the bias is a result of the choice of transverse oscillation length $\lambda_{x}$, which is determined from the 2D FFT of the PSF's.

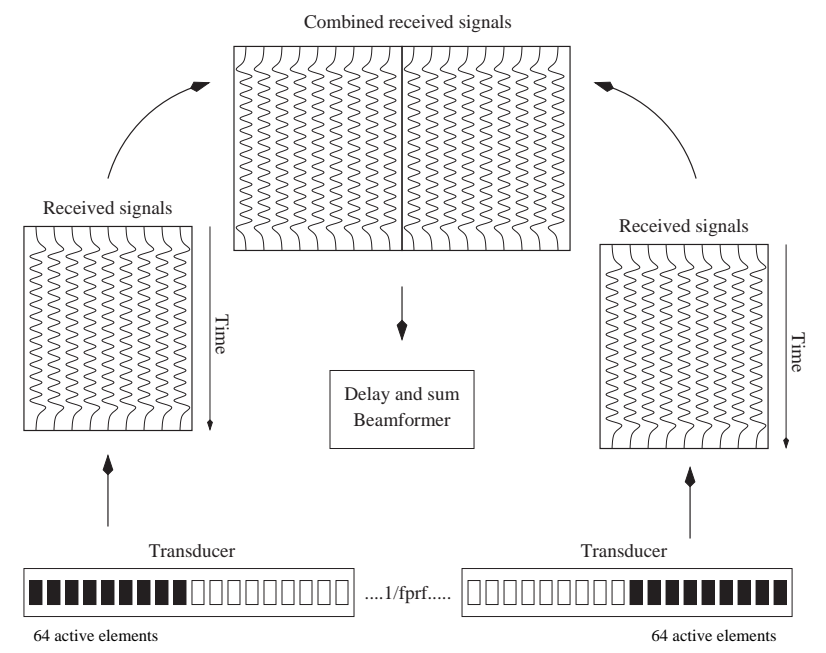

Figure 5: In order to acquire data from 128 elements in receive, signals from the left and right part of the aperture from two emissions are combined.

To demonstrate the algorithms capability of making color flow mode (CFM) images, the flow phantom was scanned using an aperture containing 64 transmitting and receiving elements, which moved across the transducer. The same trans- 
ducer setup as in the simulation was used, except for the $f_{\text {prf }}$ which was decreased to $4 \mathrm{kHz}$, in order to compensate for the reduction of the active number of transducer elements. Also the lateral wavelength $\lambda_{x}$ was increased to $\lambda_{x}=1.4 \mathrm{~mm}$. The resulting CFM image with a B mode image superimposed can be seen in Fig. 6. Only the velocities inside the vessel area have been shown. Some noise in the estimates is present, which presumably is a result of the echo canceling filter implemented.

In order to test the TO methods ability to work in dynamic conditions, the flow phantom was scanned when a pulsating flow resembling the human femoralis was present. The same setup parameters and velocity estimator was used as in the simulation, except that now the $f_{p r f}$ was set to $7 \mathrm{kHz}$. 10,000 beamformed signals were acquired, and 100 beamformed signals were used for each velocity estimation. The velocity estimates can be seen in Fig. 7. It is seen that the characteristics of the pulsatile flow can be estimated.
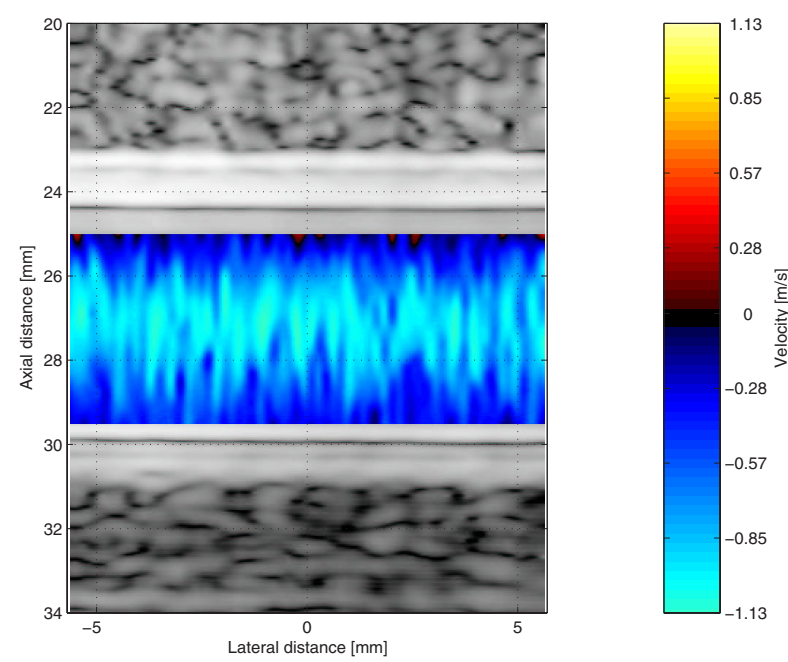

Figure 6: Transverse CFM image of the flow phantom. The dynamic range in the Bmode image is $45 \mathrm{~dB}$.

\section{CONCLUSION}

The bias and standard deviation of the measured velocities was comparable with standard deviation and bias for conventional axial Doppler estimation. Therefore, it has been demonstrated that the method can estimate the blood velocity of a purely transverse flow in simulations and in flow phantom experiments.

\section{ACKNOWLEDGMENT}

Acknowledgement: This work was supported by grant 9700883, 9700563 and 26-01-0178 from the Danish Science

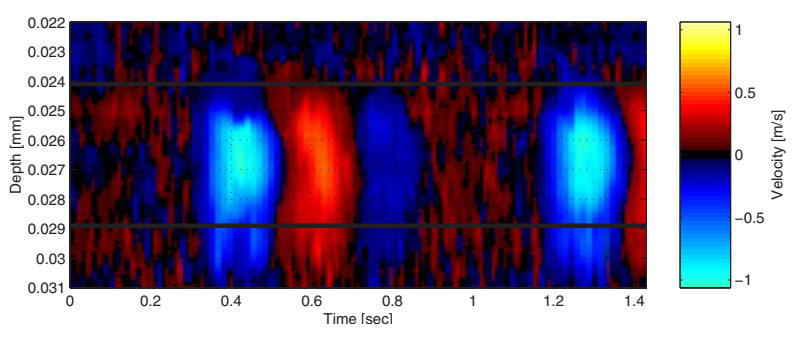

Figure 7: The velocity as a function of time for transverse flow in the flow phantom. The flow simulates the characteristics of the femoral artery. The thick lines indicate the vessel wall.

Foundation, the Ministry of Science, Technology and Development, and by B-K Medical A/S, Denmark.

\section{$\mathrm{V}$ REFERENCES}

[1] P. Munk. Estimation of the 2-D flow vector in ultrasonic imaging: a new approach. Master's thesis, Department of Information Technology, Technical University of Denmark, 1996.

[2] J. A. Jensen and P. Munk. A new method for estimation of velocity vectors. IEEE Trans. Ultrason., Ferroelec., Freq. Contr., 45:837-851, 1998.

[3] J. A. Jensen. A new estimator for vector velocity estimation. IEEE Trans. Ultrason., Ferroelec., Freq. Contr., 48(4):886-894, 2001.

[4] M. E. Anderson. Spatial quadrature: a novel technique for multi-dimensional velocity estimation. In Proc. IEEE Ultrason. Symp., volume 45, pages 1233-1238, 1997.

[5] M. E. Anderson. A heterodyning demodulation technique for spatial quadrature. In Proc. SPIE - med. imag., pages 1487-1490, 2000.

[6] J. A. Jensen. Field: A program for simulating ultrasound systems. Med. Biol. Eng. Comp., 10th Nordic-Baltic Conference on Biomedical Imaging, Vol. 4, Supplement 1, Part 1:351-353, 1996b.

[7] P. Munk. Estimation of blood velocity vectors using ultrasound. PhD thesis, Department of Information Technology, Technical University of Denmark, Lyngby, Denmark, 2000.

[8] K. V. Ramnarine, D. K. Nassiri, P. R. Hoskins, and J. Lubbers. Validation of a new blood mimicking fluid for use in Doppler flow test objects. Ultrasound Med. Biol., 24:451-459, 1998.

[9] J. A. Jensen, O. Holm, L. J. Jensen, H. Bendsen, H. M. Pedersen, K. Salomonsen, J. Hansen, and S. Nikolov. Experimental ultrasound system for real-time synthetic imaging. In Proc. IEEE Ultrason. Symp., volume 2, pages 1595-1599, 1999. 